\title{
Depth-To-Audio Sensory Substitution for Increasing the Accessibility of Virtual Environments
}

\author{
Shachar Maidenbaum ${ }^{1}$, Daniel Robert Chebat ${ }^{1,2}$, \\ Shelly Levy-Tzedek ${ }^{1,2}$, and Amir Amedi ${ }^{1,2}$ \\ ${ }^{1}$ Department of medical neurobiology, Institute for Medical Research Israel-Canada, \\ Faculty of Medicine \\ ${ }^{2}$ The Edmond and Lily Safra Center for Brain Research \\ The Hebrew University of Jerusalem, Hadassah Ein-Kerem, Jerusalem, Israel
}

amira@ekmd.huji.ac.il

\begin{abstract}
As most computerized information is visual, it is not directly accessible to the blind and visually impaired. This challenge is especially great when discussing graphical virtual environments. This is especially unfortunate as such environments hold great potential for the blind community for uses such as social interaction, online education and especially for safe mobility training from the safety and comfort of their home. While several previous attempts have increased the accessibility of these environments current tools are still far from making them properly accessible. We suggest the use of Sensory Substitution Devices (SSDs) as another step in increasing the accessibility of such environments by offering the user more raw "visual" information about the scene via other senses. Specifically, we explore here the use of a minimal-SSD based upon the EyeCane, which uses point depth distance information of a single pixel, for tasks such as virtual shape recognition and virtual navigation. We show both success and the fast learned use of this transformation by our users in these tasks, demonstrating the potential for this approach and end with a call for its addition to accessibility toolboxes.
\end{abstract}

\section{Introduction}

\subsection{Motivation}

Virtual worlds and environments are increasingly gaining importance in our lives. They can be used for a wide variety of purposes ranging from games through education, navigation and scientific research to simply general social interaction.

Yet as these virtual worlds are mainly built around visual information, they are almost completely inaccessible to the blind. This is especially unfortunate as such environments hold great potential for the blind community for uses such as social interaction and online education from the safety and comfort of their own home.

Furthermore, the main potential of these virtual environments for the visually impaired lies in the field of mobility, which poses some of their greatest everyday challenges. Since it is well established that spatial information can be transferred between virtual and real-world environments both for the general population $[1,2]$ and 
for the blind $[3,4]$, they can be used for familiarization with an environment virtually through freely available virtual worlds (for example, Google Street-View) before visiting it in the real world. This is far safer than learning to navigate through them in the real world [5], and as this can be done alone at one's own leisure, it spares the cost and availability problems of personal instructors.

\subsection{Related Work}

Unfortunately, current generic tools for making computerized graphic information accessible to the blind are still far from adequate for virtual environments.

Commonly, these tools are Text-based, such as Screen-Readers. They read the textual information to the blind user and thus make it accessible non-visually. However, such tools are simply not relevant for the transfer of graphical spatial information.

Other common tools substitute the visual information by mapping different virtual objects to text or auditory cues. This approach has birthed many attempts at creating dedicated virtual environments for the blind [see review in [6]. However, none of these attempts has graduated past the research stage as they suffer from the dual problem of interfacing the graphic information to the user and the initial creation of these tags, which involves either heavy automatic pre-processing or labor-intensive human tagging which makes them impractical on a large scale or for the existing virtual environments.

It should be noted that several tools exist to increase the accessibility of specific environments and have achieved impressive results $[7,8]$, but even they are still far from being decreed accessible.

\subsection{Sensory Substitution for Increasing Accessibility and the Virtual - EyeCane}

A different potential approach, which we utilize here, is the use of Sensory Substitution Devices (SSDs). In this approach, the visual information in the scene is transferred to the user via alternative senses. SSDs rely on the ability of the brain to reinterpret this information coming through a different sense and then transform and process it for its original task. SSDs such as the vOICe and Brainport have been used to obtain impressive results both in the real world (reviewed in [9]), and in laboratory settings (for example [10-12]). However, SSDs have several known problems limiting their wide adoption. Mainly, they require long periods of training to master their use and are hard to use in noisy real-world environments [13].

As one of the first steps upon this path we explore the use of a minimal-SSD which transfers a single point of information. Specifically, we use a virtual version of the EyeCane [14]. The EyeCane augments the traditional white cane using sensors to detect obstacles from a greater distance $(5 \mathrm{~m})$ and transforms this information into a simple auditory cue (the user hears a series of beeps, where the closer the object the user is pointing at, the higher the frequency of cues).

The Virtual-EyeCane, which we describe here, gives the same sensory output as the real-world EyeCane. This feature has the potential of easing the transfer of learning from the virtual environment to the real one. Additionally, as this simulation relies only on the distance between the participant and the object they are pointing at, it can 
be easily calculated in any 3D mesh which is the foundation of nearly every virtual environment.

We will present our recent results $[14,15]$ showing the fast-learned practical use of this algorithm by blind and blindfolded-sighted participants for navigation in simple virtual environments, and for recognizing $2 \mathrm{D}$ and 3D shapes after less than $10 \mathrm{mi}-$ nutes of training.

We will then present for the first time results showing the feasibility of using this approach to enable successful navigation in more complex virtual indoor environments including rooms and corridors with multiple decision points. Additionally, we will present new results showing the shape identification task can be performed online after self-training with comparable results to those achieved with an instructor.

\section{General Methods}

\subsection{Participants and Ethics}

The experiment was approved by the Hebrew University ethics committee, and all participants signed informed consent forms.

\subsection{Experimental Setup}

We created dedicated virtual environments using Blender 2.49, and Blender-Python modules using python 2.6.2. The environments produce a graphical output of the virtual environment. However, it was not available to the participants in this experiment, who were either blind or blindfolded. The software automatically logs any activity within the virtual environments.

The environments are controlled using a standard keyboard and a mouse.

\subsection{The Virtual EyeCane}

In the real world, the EyeCane uses a set of IR sensors to sense the distance to the object it is pointed at. This distance is then transformed to sound such that the shorter the distance the higher the frequency of sounds [14]. The Virtual EyeCane calculates the distance using Blender's standard Ray-Casting algorithm (which calculates the distance to the object the virtual device is directly pointed at, much like the sensors of the EyeCane) and links it to a sound file recorded from the EyeCane's auditory output, which the user than uses to perceive the distance. Distances within the environment are set so that each "blender meter" correlates to a real world meter so that the EyeCane and virtual-EyeCane give identical outputs at the same distance.

\section{Task 1 - Virtual Shape Recognition in the Lab and Online}

\subsection{General and Previous Work}

As a first step we tested [15] whether our participants could use this approach to recognize simple 2D \& 3D virtual shapes. This experiment was conducted with no training at all, to explore the concept's simplicity. 
We found there that participants $(n=26,23$ sighted \& 3 blind, 11 Male, aged $25.1 \pm 5.3)$ were indeed able to correctly recognize $2 \mathrm{D}$ shapes $(61.1 \% \pm 12.9 \%(\mathrm{SD})$, $\mathrm{p}<5 \mathrm{E}-9$, standard t-test. Square, triangle \& circle) and 3D shapes $(81.1 \% \pm 17.8 \%$, $\mathrm{p}<3 \mathrm{E}-13$. Pyramid, Half-Pipe, Ball \& Bowl).

The next step, reported here for the first time, was to explore the ability of users to learn this transformation by themselves without supervision from an instructor. To test this, and to expand the pool of participants, we created an online version of this experiment and opened it to public access.

\subsection{Methods}

Participants. 41 users completed this study. No additional data was collected about them.

Training. Users had access to a training task which consisted of 2 training levels and included being told the correct answer as feedback and been allowed to try again until success.

Experimental Paradigm. Both the 2D and 3D tasks each included 8 levels with 4 different shapes (see fig 1a-b). No information about the shape was presented visually on the screen, forcing them to rely on the information from the virtual-EyeCane. The experiment is online at http://brain.huji.ac.il/online_games/Default.aspx (note that the experiment and instructions are in Hebrew).
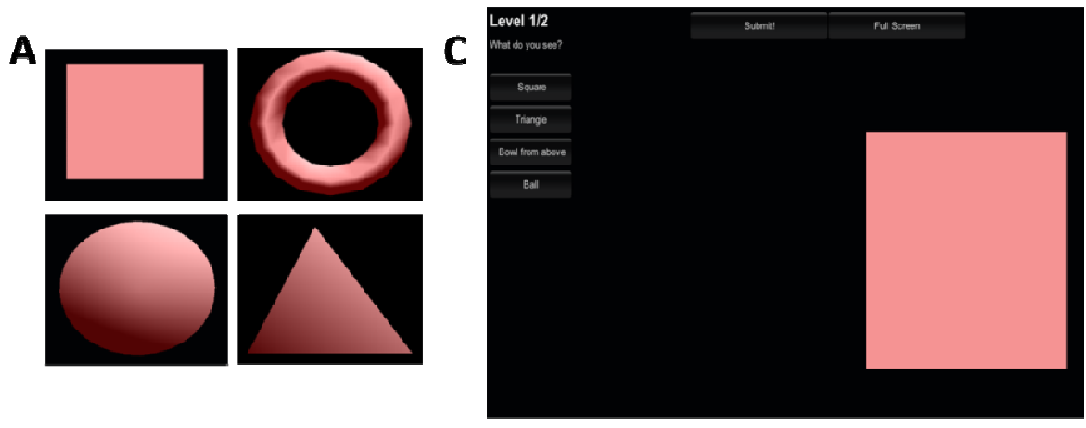

B

D
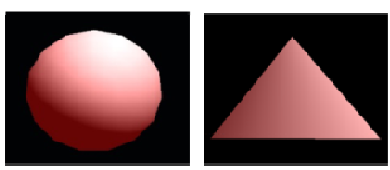

\section{Success in recognizing virtual shapes online}


Fig. 1. Online shape recognition (A) 2D shapes: Square, Circle, Ring, Triangle (B) 3D shapes: Ball, Cone, Pyramid, Cylinder (C) Screenshot of a level with the shape displayed (D) Results for shape recognition in $2 \mathrm{D}$ and $3 \mathrm{D}$ shapes. 


\subsection{Results}

Participants correctly recognized 2D shapes with a significant success rate of $63.1 \% \pm 20.3 \%(\operatorname{mean} \pm \mathrm{SD})(\mathrm{p}<5 \mathrm{E}-12$, standard $\mathrm{t}$-test $)$ and the 3D shapes with a significant success rate of $47.9 \% \pm 30.0 \%(\mathrm{p}<5 \mathrm{E}-3)$. These results fall within the range of the results reported in [15]. See graph in figure 1d.

\section{Task 2 - Simple Virtual Navigation}

\subsection{General and Previous Work}

We have recently explored [14] whether blind and blindfolded users can navigate down a virtual twisting corridor using only the Virtual-EyeCane's single point distance parameter, after only a few minutes of training. We found there that all participants ( $\mathrm{n}=23,20$ sighted $\& 3$ congenitally blind, 9 Male, Aged 27.6 \pm 8.4 ) were able to complete all levels while significantly shortening the required time, path length and number of collisions over repeated trials.

Those results showed that all participants were indeed able to navigate all routes successfully following minimal training.

However, these results were obtained in very simple environments, which consisted of twisting corridors with no decision points, a consistent structure with only 2 turns, and with constant walls spaced on both sides of the users.

Here, we expand these results to show that they hold even in more complex environments, specifically that similar minimal training is sufficient for navigating through rooms and corridors with decision points.

\subsection{Methods}

Training. Prior to the task participants travelled through 2 virtual training routes to familiarize them with the keyboard controls and environment. Participants were instructed and encouraged to experiment within them by walking towards and away from walls - so they become familiar with the auditory feedback associated with approaching a wall - and colliding with the walls while taking their time to explore the corridors. This training session lasted no more than 7 minutes and was accompanied by verbal feedback from the instructor.

Levels. This experiment included 4 corridors and 5 rooms (fig 2a-b). In the Corridors, participants were tasked with navigating to the end point which was described to them verbally (e.g. "The exit is on the $3^{\text {rd }}$ corridor on the right"). In the Rooms, participants were tasked with finding the exit with no further instructions.

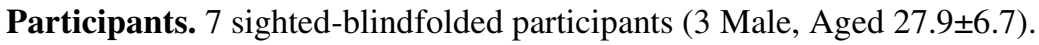

\subsection{Results}

Participants navigated successfully within the allotted timeframe with a success rate of $64.2 \pm 6.8$ for corridors and $94.2 \pm 3.4$ for rooms (figure $2 \mathrm{c}$ ). These results are significant as without any assistive device there is no way for the user to complete these mazes. 
A
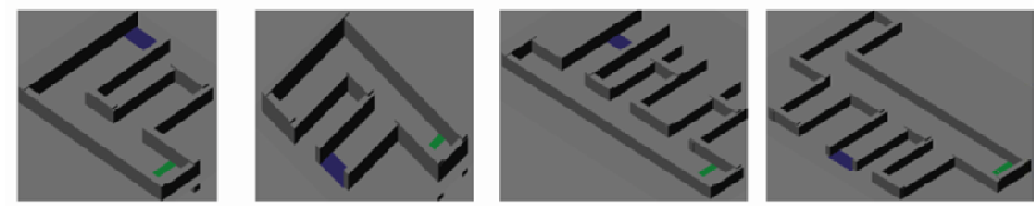

B
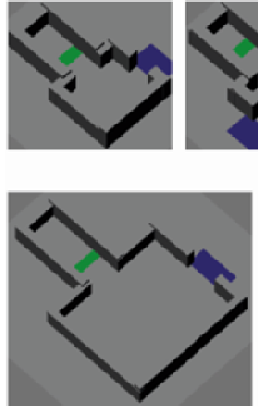
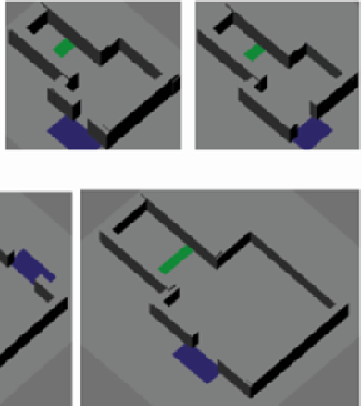
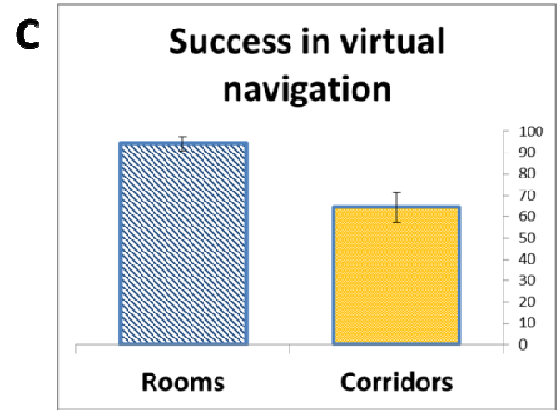

Fig. 2. Virtual navigation. (A) The virtual corridors (B) The virtual rooms (C) Results for success in the virtual navigation tasks.

\section{Discussion}

\section{$5.1 \quad$ General}

These results suggest that the approach of using virtual canes in virtual environments is both feasible and beneficial to potential users, and enables them to perform virtual tasks which are otherwise impossible for them. Our results show that even using such a simple feedback parameter as distance to an object, and even using only it, can enable the blind to perform tasks otherwise impossible to them, thus incrementally improving the accessibility of online 3D environments and objects in a way that can easily be combined with other state-of-the-art tools for accessing other types of computerized information such as screen-readers.

A key point of our method is that it is easily usable without the need to computationally pre-process a specific scene, but rather relies on data that a customized plugin to almost any virtual environment will be able to supply. Thus, instead of having to create each scene with a virtual world, the user would only need to download a plugin for the specific environment (such as WoW, Google-Earth etc.).

Another factor enhancing our method's accessibility is that it does not rely on any special equipment, but rather only on standard hardware - a sound output source (speaker/headphones) and input devices (mouse/keyboard).

This success with even a minimal-SSD indicates the potential of more complex SSDs such as the EyeMusic [16-17] or the vOICe [18] for increasing accessibility in a far more significant fashion, as they offer whole-scene information and parameters such as shape, location and color. 


\subsection{Training}

The results from the online task indicate that users can indeed train themselves with this tool, potentially enabling a solution to the cost and lack of availability of personal instructors required by other tools. It will be important to explore whether this result can indeed hold for more complex tasks and transformations.

\subsection{Gamification and Positive User Experience}

Participants treated the Virtual-EyeCane experiments as games, and often requested to continue playing with them after the experiment ended (all participants volunteered to participate in future experiments). This echoes previous successful reactions to other online game-based tools for the blind [4], and demonstrates the efficacy of the current popular trend of using gamification for general research and education in the general public.

\subsection{Lack of Vestibular and Proprioceptive Information}

One of the main difficulties users had was the lack of vestibular \& proprioceptive information as they navigated through the environments, especially when turning, which often caused confusion. This is a common problem with all virtual simulations of motion. It would be interesting to explore this question in the future using full body control instead of a keyboard interface and see the effect of such information. It should be noted that without vision such orientation is often harder than we one would expect [19].

\section{Conclusion}

We have shown here that using our method it is both feasible and simple to perform virtual tasks such as shape recognition and navigation using single-point depth information for both blind and blindfolded-sighted individuals, a task which is impossible for them without such a method.

This approach has several advantages such as simplicity and extendibility to existing virtual environments, thus increasing their accessibility to the blind. The use of identical stimuli in the virtual world from the Virtual-EyeCane to that given by the EyeCane in the real world may potentially increase the efficiency of its use in novel real-world scenarios learned virtually.

We call on further research to test whether SSD can indeed be used for enabling the visually impaired to accomplish further, more complex, tasks in virtual environments, and for making these environments more accessible in general. Additionally, we believe this ability will be extended significantly when used together with other devices such as the EyeMusic. 
Acknowledgements. We would like to thank Shlomi Hanassy and Sami Abboud for their help in developing the EyeCane, upon which the virtual EyeCane is based, Itay Ariel for his help in developing the online system and experiment, and Ori Lavi for her help in running the experiment. This work was supported by a European Research Council grant to A.A. (grant number 310809); The Charitable Gatsby Foundation; The James S. McDonnell Foundation scholar award (to AA; grant number 220020284); The Israel Science Foundation (grant number ISF 1684/08); The Edmond and Lily Safra Center for Brain Sciences (ELSC) Vision center grant (to AA) and an ELSC fellowship (to SL \& DRC); The Azrieli Foundation fellowship award (to DRC).

\section{References}

1. Witmer, B.G., et al.: Virtual spaces and real world places: transfer of route knowledge. International Journal of Human-Computer Studies 45(4), 413-428 (1996)

2. Foreman, N., et al.: Spatial information transfer from virtual to real versions of the Kiel locomotor maze. Behavioural Brain Research 112(1), 53-61 (2000)

3. Lahav, O., Mioduser, D.: Exploration of unknown spaces by people who are blind using a multi-sensory virtual environment. Journal of Special Education Technology 19, 15-24 (2004)

4. Merabet, L.B., et al.: Teaching the Blind to Find Their Way by Playing Video Games. PloS One 7(9), e44958 (2012)

5. Manduchi, R., Kurniawan, S.: Watch Your Head, Mind Your Step: Mobility-Related Accidents Experienced by People with Visual Impairment. Tech. Rep. UCSC-SOE-10-24, University of California, Santa Cruz (2010)

6. Lahav, O.: Improving orientation and mobility skills through virtual environment for people who are blind: past research and future potential. In: ICDVRAT 2012, Laval (2012)

7. White, G.R., Fitzpatrick, G., McAllister, G.: Toward accessible 3D virtual environments for the blind and visually impaired. In: Proceedings of the 3rd International Conference on Digital Interactive Media in Entertainment and Arts, pp. 134-141. ACM (September 2008)

8. Trewin, S., Hanson, V.L., Laff, M.R., Cavender, A.: PowerUp: an accessible virtual world. In: Proceedings of the 10th International ACM SIGACCESS Conference on Computers and Accessibility, pp. 177-184. ACM (October 2008)

9. Maidenbaum, S., Amedi, A.: Applying plasticity to visual rehabilitation in adulthood. In: Jennifer Steeves, L.R.H. (ed.) Plasticity in Sensory Systems. Cambridge University Press (2012)

10. Striem-Amit, E., Guendelman, M., Amedi, A.: 'Visual' acuity of the congenitally blind using visual-to-auditory sensory substitution. PloS 1 (2012)

11. Chebat, D.R., Schneider, F.C., Kupers, R., Ptito, M.: Navigation with a sensory substitution device in congenitally blind individuals. Neuroreport 22(7), 342-347 (2011)

12. Chebat, D.R., Rainville, C., Kupers, R., Ptito, M.: Tactile-'visual' acuity of the tongue in early blind individuals. Neuroreport 18(18), 1901-1904 (2007)

13. Reich, L., Maidenbaum, S., Amedi, A.: The brain as a flexible task machine: implications for visual rehabilitation using noninvasive vs. invasive approaches. Current Opinion in Neurology (2011)

14. Maidenbaum, S., et al.: Increasing Accessibility to the Blind of Virtual Environments, Using a Virtual Mobility Aid Based On the EyeCane. PloS One 8(8), e72555 (2013) 
15. Maidenbaum, S., et al.: Virtual 3D shape and orientation discrimination using point distance information (2012)

16. Abboud, S., Hanassy, S., Levy-Tzedek, S., Maidenbaum, S., Amedi, A.: EyeMusic: Introducing a "visual" colorful experience for the blind using auditory sensory substitution. Restorative Neurology and Neuroscience (2014)

17. Levy-Tzedek, S., Novick, I., Arbel, R., Abboud, S., Maidenbaum, S., Vaadia, E., Amedi, A.: Cross-sensory transfer of sensory-motor information: visuomotor learn (2012)

18. Meijer, P.B.: An experimental system for auditory image representations. IEEE Transactions on Biomedical Engineering 39(2), 112-121 (1992)

19. Loomis, J.M., Klatzky, R.L., Giudice, N.A.: Representing 3D space in working memory: Spatial images from vision, hearing, touch, and language. In: Multisensory Imagery, pp. 131-155. Springer, New York (2013) 